\title{
Thermal Analysis by Structural Characterization (TASC): Structural and Thermo-Rheological Information from Hot Stage Microscopy
}

\author{
M. Reading \\ Department of Pharmacy, University of Huddersfield, Queensgate, Huddersfield HD1 3DH, UK \\ m.reading@hud.ac.uk
}

\begin{abstract}
A new technique for thermal analysis has recently been introduced based on hot stage microscopy and software for image analysis called thermal analysis by structural characterization or TASC. It enables new measurements to be made: for a range of materials, including polymers and pharmaceuticals, we describe measuring glass transitions using 3D imaging based on z-stacking, characterizing complex melting behavior where there is a distribution of melting points, and thermo-mechanical analysis with $2 \mathrm{D}$ detection. In this way the range of measurements available to users of hot stage microscopy has been expanded.
\end{abstract}

\section{Introduction}

Thermal methods are commonly used to characterize a wide range of materials. Measuring transition temperatures can provide a wealth of information about the structure and properties of polymers, pharmaceuticals, and ceramics. However, the most commonly used techniques for measuring glass and melting transitions-differential scanning calorimetry (DSC), thermomechanical analysis (TMA), and dynamic mechanical analysis (DMA) - provide only global information averaged over the whole sample. Local information about small areas isn't available. Furthermore, the smallest samples are of the order of milligrams in the case of DSC and typically grams in the cases of TMA and DMA. The introduction of micro/nano thermal analysis based on atomic force microscopy made possible, for the first time, the measurement of transition temperatures in a localized way as well as measurements on nanoscale objects [1]. Whilst this represented an advance, atomic force microscopes are expensive and relatively difficult to use. The largest field of view is frequently only $100 \mu \mathrm{m}^{2}$, and samples must be smooth because the maximum $z$ displacement is typically $10 \mu \mathrm{m}$ or less.

Hot stage microscopy (HSM), also called thermomicroscopy, is a well-established method that can examine $\mu \mathrm{m}$-sized to $\mathrm{mm}$-sized objects and is frequently applied to ordered materials, such as liquid crystals. The method struggles, however, with amorphous samples as well as many opaque samples. Transmitted or reflected light intensity measured as a function of time and temperature can be useful, but, once again, opaque samples are challenging. There is usually no intuitive relationship between light intensity and the sample property being measured; for example, melting could result in an increase or decrease

in light intensity depending on the background, lighting, and the optical properties of the sample.

We have developed a method called thermal analysis by structural characterization (TASC), which directly measures changes in structure observed in a light optical microscope with a temperature-controlled stage [2,3,4]. It is a method of image analysis that is universal in that it does not depend on the presence of specific types of structures. A crucial capability is that it removes the effect of sample movement. When a sample is heated it usually moves due to its thermal expansion and/or the expansion of the sample chamber. By removing this effect the system algorithm returns values related only to structural change. The details of how this is achieved have been given elsewhere [3]. It has been shown that TASC provides a means of: (a) measuring local transition temperatures, (b) analyzing very small samples (down to a few micrograms), (c) quantifying heterogeneity, and (d) characterizing dissolution behavior.

In this article we discuss for the first time how TASC can be used to look at glass transitions using $3 \mathrm{D}$ imaging. We also present for the first time an image based on transition temperatures measured using TASC (called T-Map mode) as part of a study of complex melting behavior. We also describe a TASC-related method to create a form of thermomechanical analysis within a hot stage microscope.
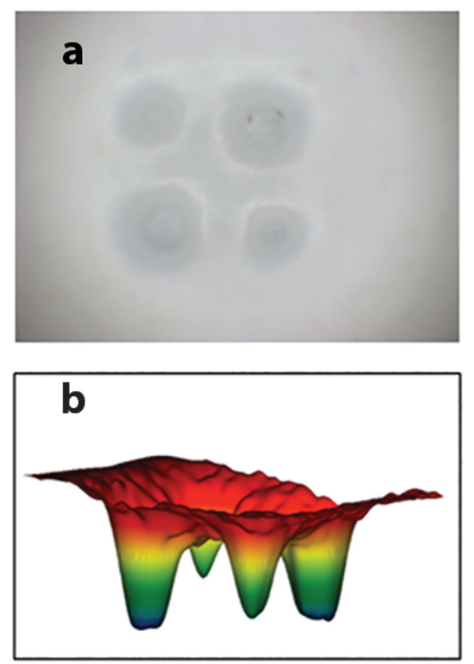

Figure 1: Relaxation of filled polystyrene. (a) Light microscope image of a pattern of four indentations in the sample of filled polystyrene. (b) 3D image of surface topography obtained with extended depth of field derived from z-stacking of multiple images. (c) Thermal analysis by structural characterization (TASC) plot with the topography maps from points along the relaxation process. 


\section{Incomparable...}

...and still innovating

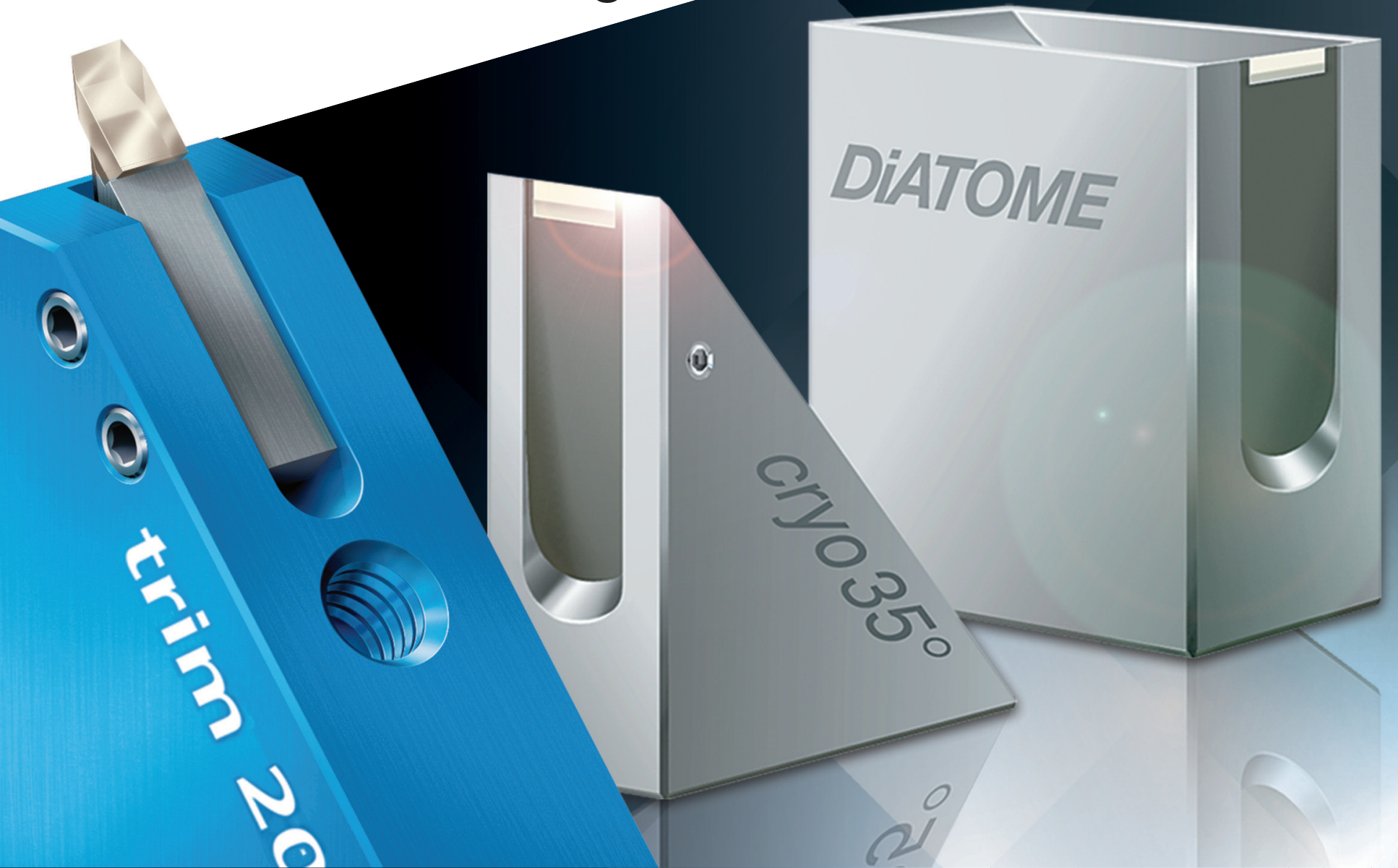

\section{DIATOME diamond knives}

ultra $45^{\circ} \cdot$ cryo $\cdot$ histo ultra $35^{\circ}$ - histo jumbo cryo immuno • ultra sonic ultra AFM \& cryo AFM trimtool 20 - trimtool 45 trimtool 90

Over 40 years of development, manufacturing, and customer service

\section{DIATOME U.S.}

P.O. Box $550 \bullet 1560$ Industry Rd. • Hatfield, Pa 19440 Tel: (215) 412-8390 • Fax: (215) 412-8450

email: sgkcck@aol.com • www.emsdiasum.com
Free customer service

Sectioning tests with biological and material research specimens of all kinds. We send you the sections along with the surfaced sample, a report on the results obtained and a recommendation of a suitable knife. Complete discretion when working with proprietary samples.

Re-sharpening and reworking service

A re-sharpened Diatome diamond knife demonstrates the same high quality as a new knife. Even knives purchased in previous years can continue to be re-sharpened. The knives can be reworked into another type of knife for no extra charge, e.g. ultra to cryo or $45^{\circ}$ to $35^{\circ}$.

\section{Exchange service}

Whenever you exchange a knife we offer you a new DiATOME knife at an advantageous price. 


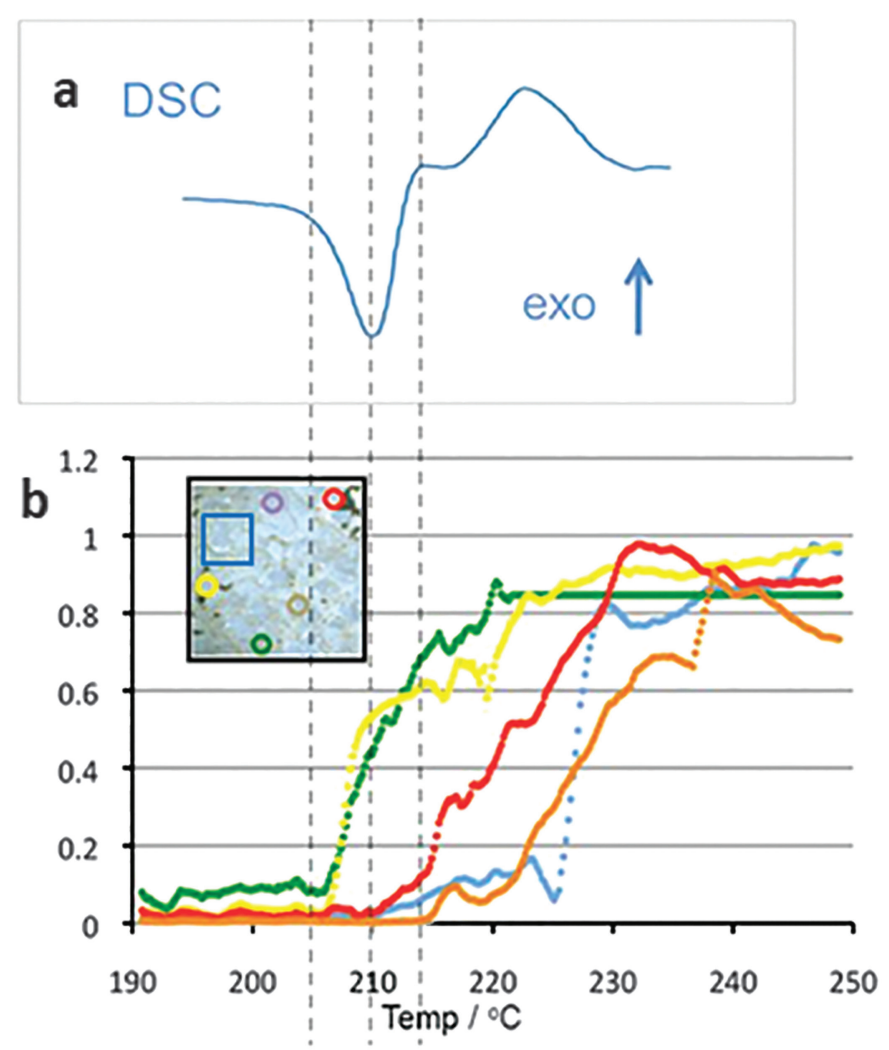

Figure 2: Melting of zoledronic acid. (a) DSC plot showing an onset temperature of $205{ }^{\circ} \mathrm{C}$, peak temperature of $210^{\circ} \mathrm{C}$, and offset temperature of $217^{\circ} \mathrm{C}$. The endotherm is followed by an exotherm attributable to a decomposition's reaction. (b) The TASC graph shows this complex behavior where the melting continues during the exotherm. The TASC graph follows the color coding given by the circles in the inset micrograph showing the locations of the individual measurements. Image width $=2000 \mu \mathrm{m}$. The blue box indicates the area shown in Figure 4 .

\section{Materials and Methods}

The apparatus was a Linkam THMS 600 Stage with Imaging Station (Linkam Scientific Instruments, UK) controlled by Linksys software with the TASC option. The polymer sample was polystyrene filled with calcium carbonate called StripStyrene (Evergreen Corp., USA). The 3D images were acquired using a Zeescan (Phaseview, France) fitted to the Linkam Imaging Station. The zoledronic acid was from Gador Laboratory (Gador S.A., Argentina). The DSC was a prototype dual-cell DSC from Linkam. The TMA probe was a prototype made by Cyversa Ltd (UK). The polycaprolactone (PCL) was from Vornia Biomaterials (Ireland).

In a typical TASC experiment a series of images is collected over time as the temperature is raised at a near-constant rate. These images are then analyzed retrospectively by selecting an area with the curser, called the region of interest (ROI), within which is located the structure of interest. A second area is then selected around the first, called the scanned area, and this represents the area within which the structure of interest is allowed to move. If there is no movement these two areas could be the same but, at high magnification, some movement will almost always occur. The amount of movement can be assessed simply by inspection of the collected micrographs. Because the actual TASC value depends on the number of pixels and light intensity, among other variables, the TASC curve is normalized. Where detailed comparisons are being made between samples, care must be taken to ensure the lighting and magnification are the same. This is usually not difficult to achieve because the user can refer to previous images and adjust these variables appropriately. In one of the cases described below, a whole series of TASC measurements was made by dividing the field of view into a grid. Each square became a scanned area with the ROI being $10 \%$ less in $x$ and $y$. The results for the transition temperatures were obtained manually and presented in 3D using Matlab ${ }^{\circledR}$ (MathWorks, USA). Although the data were obtained manually in this case, it can be envisaged how this process could be automated.

As part of the process for measuring glass transitions, an indentation was made in the surface of the sample. The procedure was to raise the sample temperature above its glass transition, place the tool on the surface, cool the sample to below its glass transition, and then remove the tool. In this way there was no plastic deformation or frozen-in stress; thus the measurement was purely a relaxation event driven by surface tension.

\section{Results}

3D Measurements of glass transitions. The filled polystyrene used in this experiment provides an example of how TASC can make measurements on opaque samples. In this case a structure,

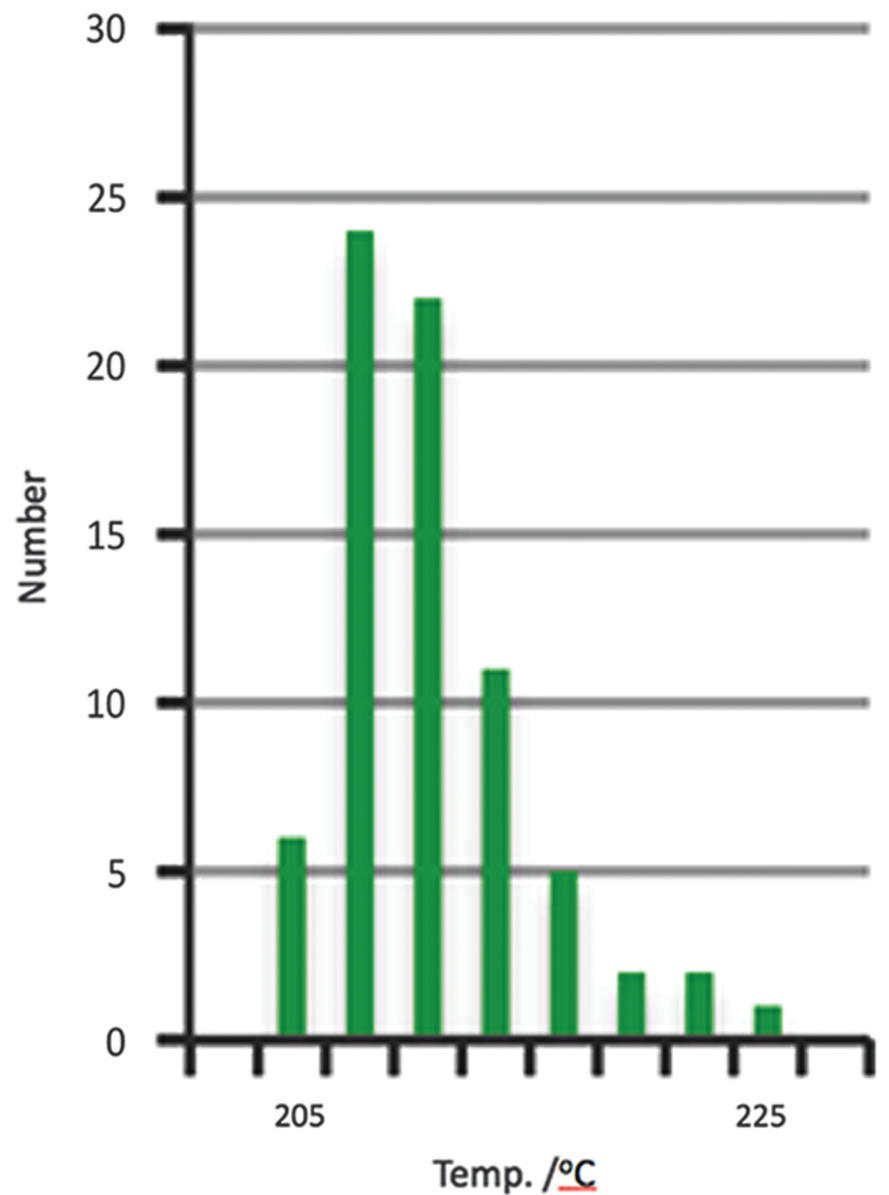

Figure 3: Data from Figure 2 can be used to create a histogram of the distribution of transition temperatures. This characterizes the melting process and can be compared with the DSC data to show that the melting endotherm is distorted by the exotherm resulting from decomposition. 


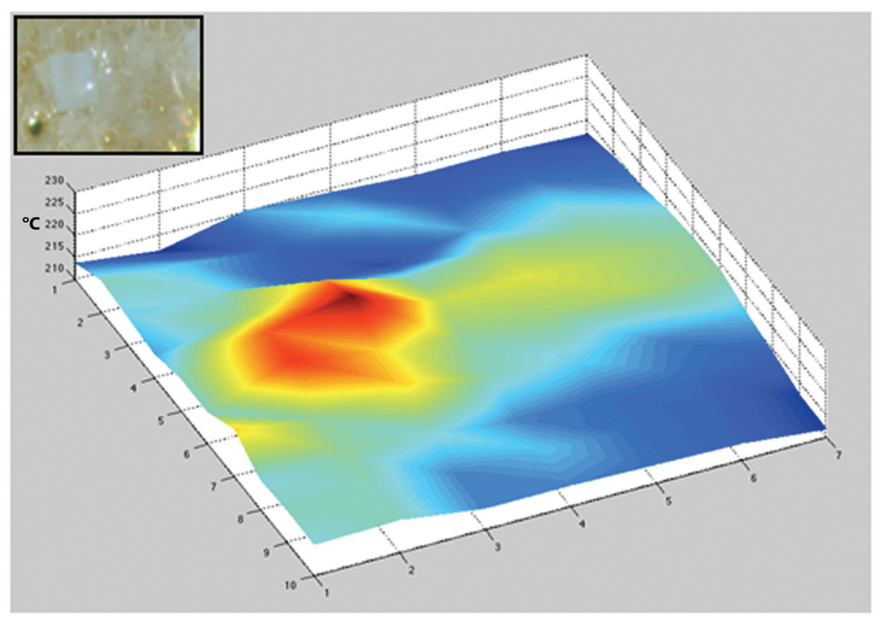

Figure 4: 3D image created by mapping transition temperatures within the region shown in the inset micrograph. Image width $=500 \mu \mathrm{m}$. The micrograph shows an unmelted crystal surrounded by bubbling molten material from crystals that melted at lower temperatures. The bubbling is due to decomposition, and the DSC trace shows an exotherm at this point. In the 3D image this crystal appears in red because of its higher melting temperature. This illustrates the inhomogeneous nature of the sample.

a series of indentations made with a custom tool, is created by the experimenter. Without this structure no measurement could be made because the appearance of the sample would not significantly change as it softened. Because of the indentations, the transition can be detected as a consequence of the fact that, at the glass transition temperature, the material becomes fluid and surface tension causes the material to flow thereby making the indentations disappear [2]. Images in 2D can provide satisfactory results for detecting transition temperatures as shown here and elsewhere $[2,3,4]$. Measurements of the kinetics of glass transitions made to date have used 2D images; this is acceptable because isoconversion points can still be identified [2], but the volume of material that has flowed and the depth of the indentation would not be known. A more rigorous approach is to acquire $3 \mathrm{D}$ images of the surface. In this case topographic images were acquired by $z$-stacking. A series of micrographs was taken as the focal plane of the microscope is moved up and down. Sophisticated software enables the focused regions in each image to be extracted, and a composite image is created in which all of the parts are in focus. This is a well-established method in conventional photography and, increasingly, in microscopy.

From a $z$-stack of images, a $3 \mathrm{D}$ map of the topography of the sample can be constructed as shown in Figure 1. The disappearance of the indentations with increasing temperature can be seen in the surface images associated with the graph of Figure 1c. The TASC algorithm can equally well be applied to these topographic data, however, the process of $z$-stacking is more time-consuming than simply acquiring single $2 \mathrm{D}$ images. Thus very rapid experiments would be precluded.

In Figure 1c the topography plots are shown next to the standard 2D TASC curve and can serve as a means of calibrating $2 \mathrm{D}$ data so that, for example, the depths of the indentations can be calculated. This works where the conditions under which the 3D images were acquired are identical to those under which the 2D TASC results were obtained. In this way the TASC technique can be used to obtain more quantitative measurements of the rheological behavior of the sample.

Analyzing complex melting. Figure $2 \mathrm{a}$ shows the DSC curve for zoledronic acid. There is an initial dehydration step (not shown) [5], and then the DSC curve shows there is an endotherm followed by an exotherm. The HSM data show that the sample starts as a white solid, and then a bubbling liquid is formed that coexists with some remaining solid material (inset in Figure 2) during the mid-stage of the process. The picture that emerges is a complex one where melting and decomposition occur over a wide temperature range. This is confirmed by the TASC data shown in Figure 2b. Different places in the sample melt at different temperatures over a range of $205^{\circ} \mathrm{C}$ to $225^{\circ} \mathrm{C}$, that is during both the endotherm and the exotherm. This complexity is illustrated by the temperature map image shown in Figure $2 b$ (inset). The area shown in the inset image was divided into a $10 \times 10$ grid, and in each cell within the grid a TASC analysis was carried out. This image then gives rise to the histogram presented in Figure 3, which characterizes the heterogeneous nature of the melting/decomposition process. It means that the HSM data can be extended beyond qualitative observations. When the sample is distributed to an approximately even depth, the histogram enables the fraction of the sample that melts to be estimated as a function of temperature.

Figure 4 shows the onset temperatures of the transitions obtained from these measurements plotted in the form of a $3 \mathrm{D}$ image. We believe it is the first time this type of transition temperature map has been acquired using light microscopy. It can be seen that there is a large particle, colored red in the image, that melts significantly later (higher temperature) than the surrounding material as the temperature is gradually raised.
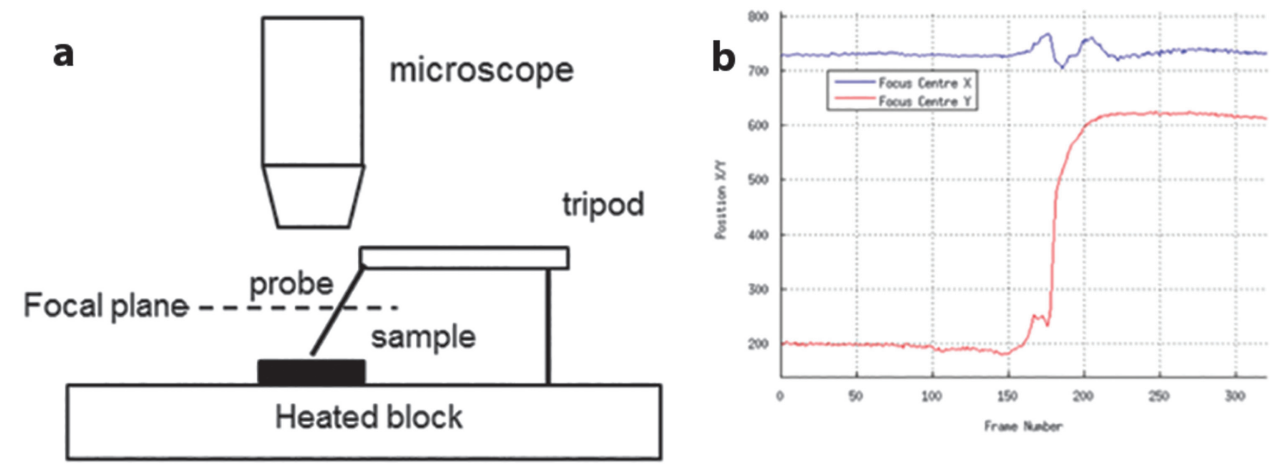

Figure 5: Thermomechanical analysis (TMA) measurements of polycaprolactone (PCL) using a simple tripod device. (a) Schematic of tripod with one leg resting on a sample placed on a heated block. The microscope focal plane is set to be along the mid-point of the probe. When the sample softens, the probe moves downward and the part of the probe that is in focus moves toward the right. Movement in the orthogonal direction is also tracked. (b) The red curve shows movement corresponding to indentation, and the blue curve shows movement sideways. As the PCL softened, the tip translated toward the side of the particle, and both $x$ and $y$ movements were observed. 
The wide range of temperatures for the transition from solid to liquid requires comment. The dehydration step probably results in significant disruption of the crystalline structure which, in turn, affects melting temperatures. Different levels of disruption occur as a consequence of differences in local conditions including sample thickness (which affects local concentrations of the evolved gas during dehydration). This would produce a wide range of temperatures at which particles transition from a significantly disordered solid to liquid. Parallel melting and decomposition is not uncommon for organic materials and is seen in this case from the DSC curve. There are, therefore, two sources of structural disruption: the dehydration step and the nucleation and growth of decomposition product. Both contribute to the melting process occurring over a broad range. The DSC data and the TASC data are highly complementary. The former shows how the energetics of the reactions in the sample change so radically that it is clear decomposition is occurring. The TASC software shows quantitatively that melting continues well into the exotherm so the areas under the endotherm and exothermal cannot be taken in isolation.

Thermomechanical Analysis. The TMA capability is achieved by placing a tripod on the hot zone of the stage with one leg (the probe) on the sample and the other two on the heated block. Figure 5 shows this setup and a set of measurements on polycaprolactone (PCL). The microscope is first used to position where the point of the probe rests on the sample, then the focal plane is raised to the midpoint of the probe. Because it is angled, as it moves up and down, the position where it is in focus shifts laterally. In contrast to the TASC measurements described above, it is this movement that is tracked, not structural change, so expansion and indentation can be measured. A special feature of this measurement is that both $x$ and $y$ movements are tracked, something not possible with conventional TMA instruments. The results for an experiment on a particle of polycapraloactone are given. The $x$ and $y$ information could be used to characterize, for example, asymmetric frozen-in stress in a stretched polymer film.

\section{Discussion}

The spatial resolution of TASC can be estimated to be about $10 \mu \mathrm{m}$. The object that is being studied must exhibit sufficient structure so that any change can be clearly detected.

The theory of the TASC method has been given elsewhere [2]; briefly the following equation obtains

$$
\tau=v R /(\lambda C)
$$

where $\tau$ is the time constant for the relaxation process, $\lambda$ is viscosity, $v$ is surface tension, and $\mathrm{R}$ and $\mathrm{C}$ are constants related to the size and geometry of the indentation and the sample. Preliminary results for measuring the kinetics of the glass transition using multiple heating rates have already been given [2].

A significant advantage of TASC for making these types of measurements is that both very slow and very fast heating rates can be used. Slow heating rates can be used because, unlike differential scanning calorimetry, the sensitivity of the measurement is unaffected by the duration of the experiment. Unlike TMA, very thin samples and a small heated chamber can be used; thus, fast heating rates don't cause large temperature gradients. This ability to cover a very large dynamic range-almost five orders of magnitude has already been demonstrated [2] — means the kinetics can be studied in more detail than with other approaches, so the deviation from Arrhenius behavior can be clearly seen [2].
By using $z$-stacking, this type of experiment could be extended using materials with known viscosity and surface tension, and the structural constants $\mathrm{R}$ and $\mathrm{C}$ could be determined. This opens up the possibility of new types of measurements where the surface tension is known, possibly from modeling: local viscosity could be determined, and, where the viscosity is known possibly from a separate measurement using a rheometer, local surface tensions could be measured. In this way the range of localized information hot stage microscopy can provide would be extended. Future work will explore these further.

\section{Conclusion}

The information available from hot stage microscopy has been substantially increased. The types of transitions that can be detected have been expanded to include local glass transitions, and the range of materials that can be analyzed has been increased to include opaque samples. Thermal analysis images can now be obtained based on transition temperatures, and thermal analysis with $3 \mathrm{D}$ images has been demonstrated. Finally mechanical measurements can be made. This is possible using a relatively inexpensive instrument that may also serve other functions.

\section{References}

[1] X Dai et al., Adv Drug Del Rev 64 (2012) 449-60.

[2] M Reading et al., in Microscopy Advances in Scientific Research and Education Vol. 2, ed. A Mendez-Villas, Formatex Research Centre, Badajoz, Spain, (2010) 1083-89. [3] M Alhijjaj et al., Anal Chem 87(21) (2015) 10848-55.

[4] M Alhijjaj et al., Pharmaceut Res 34(5) (2017) 971-89.

[5] R Riscica et al., J Pharm Sci 99(12) (2010) 4962-72.

\section{Refurbishing or servicing an electron microscope? Scope us out for your ion pump needs.}

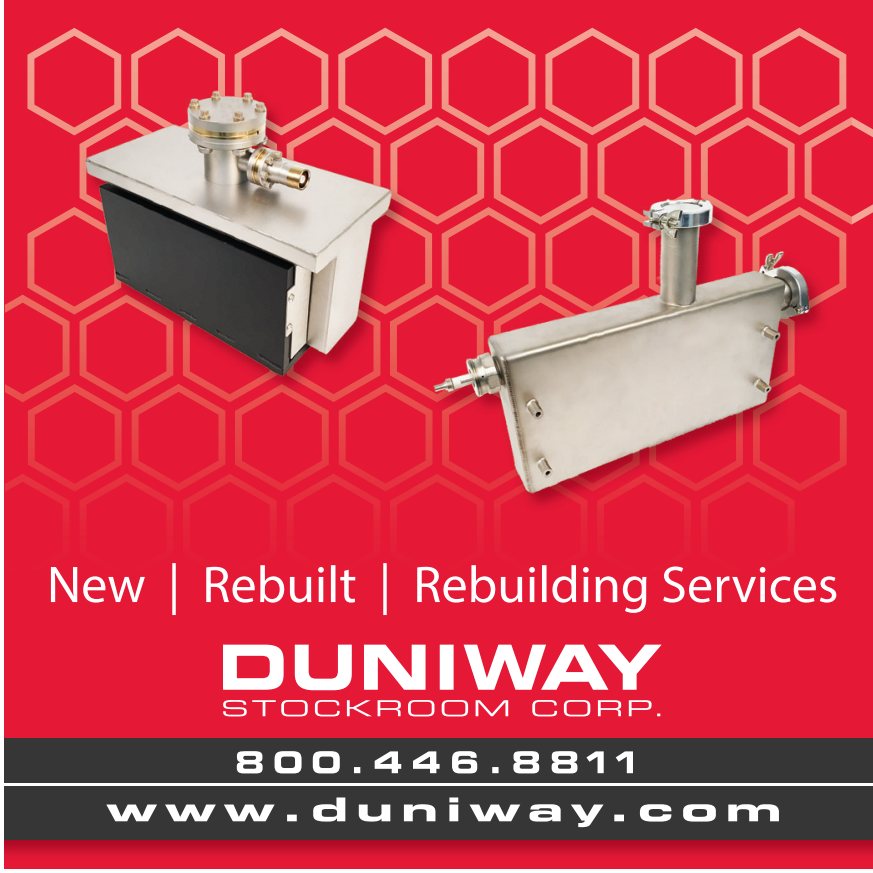




\section{TESCAN S8000G}

\section{and novel Orage ${ }^{\text {TM }}$ FIB column}

Anticipating the future, expanding your possibilities today

\section{Orage $^{\mathrm{TM}}$ FIB column: Prepare for the new level in sample preparation}

- Next generation of Ga source FIB column and a guarantee of world-class quality in sample preparation

- Cutting-edge ion beam optics delivering improved resolution at low beam energies for damage-free ultra-thin TEM specimens

- High ion beam currents up to $100 \mathrm{nA}$ enabling fast sputtering rates for maximum throughput and minimum time-to-result
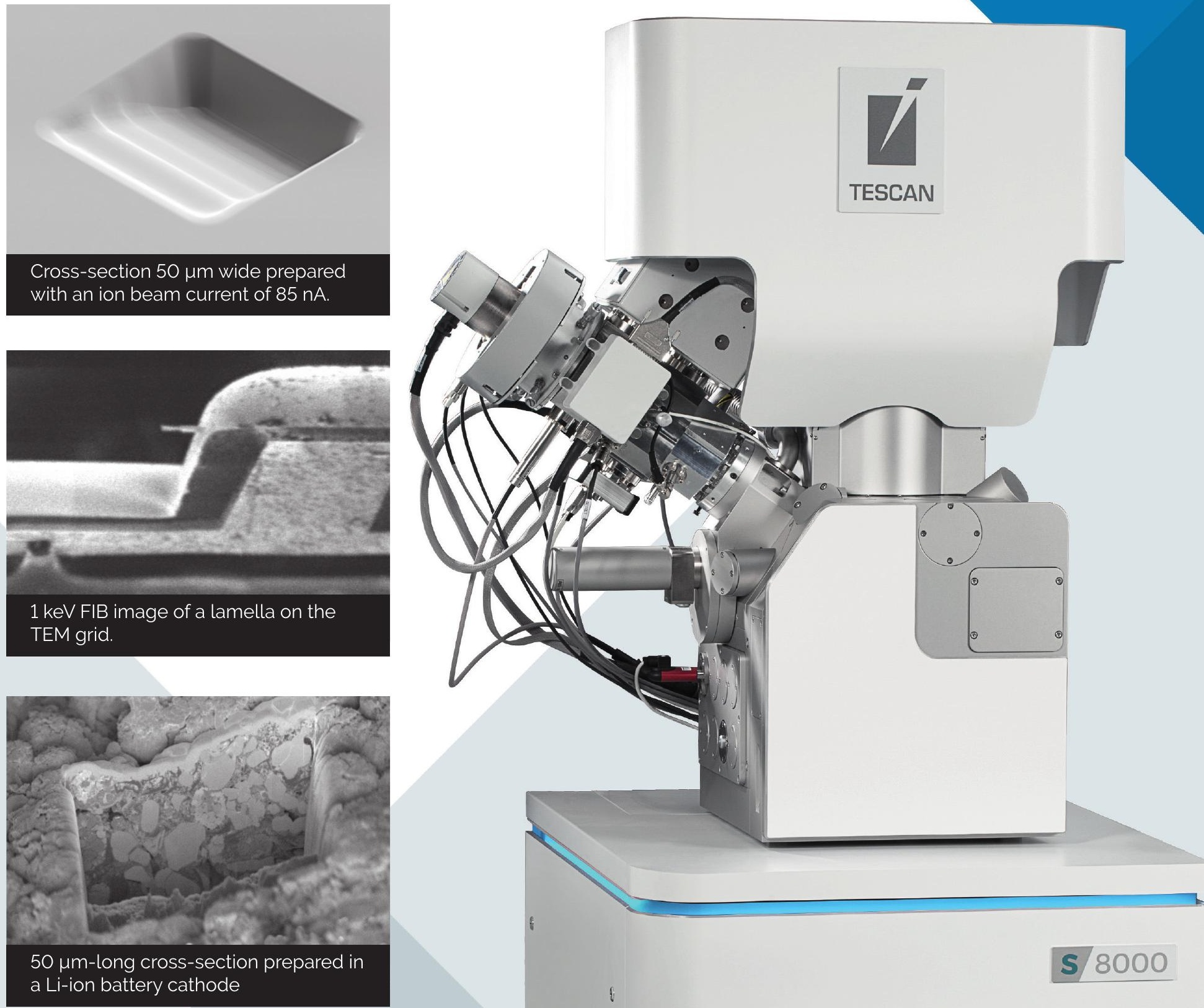